\title{
The Sucrose Starvation Signal Mediates Induction of Autophagy- and Amino Acid Catabolism-Related Genes in Cowpea Seedling
}

\author{
Ayami Kaneko $^{1}$, Eri Noguchi ${ }^{2}$, Yushi Ishibashi ${ }^{3}$, Takashi Yuasa ${ }^{3}$, Mari Iwaya-Inoue ${ }^{3}$ \\ ${ }^{1}$ Graduate School of Bioresource and Bioenvironmental Sciences, Kyushu University, Fukuoka, Japan; ${ }^{2}$ School of Agriculture, Kyu- \\ shu University, Fukuoka, Japan; ${ }^{3}$ Department of Bioresource Sciences, Faculty of Agriculture, Kyushu University, Fukuoka, Japan. \\ Email: yuasa@agr.kyushu-u.ac.jp
}

Received January $7^{\text {th }}, 2013$; revised February $15^{\text {th }}, 2013$; accepted February $28^{\text {th }}, 2013$

\begin{abstract}
In higher plants, autophagy is bulk degradation process in vacuole necessary for survival under nutrient-limited conditions and plays important roles in senescence, development and pathogenic response, etc. Cowpea is one of the most important legume crops in semi-aride region, which is highly tolerant to drought stress. Changes of photoassimilate status by drought stress and/or sink-source balance appeared to affect autophagy and senescence of leaf in cowpea. Accordingly, we focused on roles of sucrose signal in autophagy and amino acid recycling in cowpea. Effects of starvation stress on the expression of autophagy-related genes $(A T G s)$ and amino acid catabolism-related genes in cowpea [Vigna unguiculata (L.) Walp] were examined by Reverse transcription-polymerase chain reaction (RT-PCR) and anti-ATG8i specific antibody. Sucrose starvation stress enhanced the expression levels of VuATG8i, VuATG8c and VuATG4 in cowpea seedlings. The expressions of amino acid catabolism related genes, such as asparagine synthase (VuASN1), proline dehydrogenase 1 (VuProDH) and branched chain amino acid transaminase (VuBCAT2), are also up-regulated under the sucrose starvation. In contrast, high sucrose condition suppressed autophagy and the expressions of ATGs. These results indicate that sucrose starvation stress stimulates both autophagy and amino acid catabolism by regulation of $A T G s$ and $V u B C A T 2$. It is conceivable that sucrose starvation stress enhances autophagy in cowpea, possibly via branched chain amino acid level regulated by the starvation-induced BCAT.
\end{abstract}

Keywords: Amino Acid Catabolism; ATG; Autophagy; BCAT; Cowpea; Starvation Stress

\section{Introduction}

Recent studies revealed that higher plants have various pathways of protein degradation systems such as ubiqutin-proteasome, autophagy, and vacuolar processing enzyme cascade [1,2]. Among them, autophagy is nonspecific and bulk protein degradation system of intracellular components and is induced under nutrient starvation. Molecular genetic and biochemical studies with autophagy-deficient mutants of budding yeast have unveiled a set of novel genes, autophagy related genes $(A T G \mathrm{~s})$ [3]. Among $A T G$ proteins, $A T G 8$ is an ubiquitin-like protein that is first processed by the cysteine protease ATG4, removing the carboxyl terminal arginine residue and exposing a glycine residue at the carboxyl terminal end [4]. Through an ubiquitin conjugation-like mechanism, processed ATG8 is covalently conjugated to phosphatidyl ethanolamine (PE) on an autophagosome membrane [5]. Molecular genetic studies using mutants of autophagyrelated genes $(A T G S)$ unveiled that autophagy plays es- sential roles in growth, senescence, nutrient translocation and stress responses of higher plants [2,3]. In yeast and animal cells, autophagy is negatively regulated by a nutrient sensor kinase, target of rapamycin (TOR), which is stimulated by branched amino acids and by AMP-regulated kinase (AMPK) which is a plant sucrose non-fermenting 1-related kinase (SnRK) homolog involved in sugar sensing [6]. It has been shown that the conserved TOR and SnRK signaling pathways in higher plants play important roles in growth, metabolisms and stress responses including autophagy in response to nutrient and enegy levels [7].

In contrast to budding yeast, which has a single $A T G 8$ gene, the Arabidopsis thaliana genome encodes nine ATG8 (AtATG8a-i) orthologs [8]. The expressions of $A T G 8 s$ and $A T G 4$ are enhanced under sucrose starvation in plants, possibly generating intracellular amino acid pools and bioenergentic resource [9]. It is well known that status of nutrient conditions have pleiotropic effects 
on stress-related phytohormonal signals [10]. Our previous study indicated that the expression of $G m A T G 8 \mathrm{c}$ and GmATG8i in soybean at seed maturation stage transiently increased in senescing leaf at pod-filling stage [11]. At the late pod-filling stage, increases in nutrient-up take capacity and synthesis of storage proteins of developing seed possibly exert unknown inter- and intracellular signals to the leaf, leading to acceleration of autophagy and senescence, possibly via nutrient sensing of carbohydrates and nitrogen fluxes from sink organ to source organ. These observations suggest that the autophagy system in plants is regulated in more complex manner than in budding yeast.

Cowpea [Vigna unguiculata (L.) Walp] is one of major legume crop widely grown in semi-arid regions of north Africa, which has significant tolerance to drought stress [12]. Previous studies revealed that drought stress on cowpea at the seed filling stages accelerated both leaf senescence and seed maturation by enhancing nitrogen and carbohydrates translocation from senescing leaves to developing seeds [13]. These observations suggest that cowpea has significantly efficient systems of protein degradation in leaves and nutrient translocation to seeds. Resultantly, cowpea cultivation shows little or marginal reduction of the seed yield even under drought stress, compared to well-watered condition [14]. However, regulation system of autophagy and amino acid metabolism in cowpea leaves under drought stress are not yet clarified. As a candidate factor regulating autophagy and senescence, we focused on sucrose signal, which is affected by sink-source balance and reduction of photoassimilate under drought stress. Thus, a set of cowpea $A T G$ homologs, VuATG8s and VuATG4, were analyzed to address the relationships of autophagy and amino acid catabolism of cowpea in response to the sucrose starvation condition. Furthermore, it was examined whether sucrose starvation enhanced the expression of VuBCAT2 involved in intracellular BCAA levels which possibly regulates autophagy induction via target of rapamycin (TOR) cascade.

\section{Materials and Methods}

\subsection{Plant Materials}

Seeds of cowpea (Vigna unguiculata (L.) Walp. cv. IT98K-205-8) were sown in the sand filled in plastic box $\left(16 \mathrm{~cm}\right.$ side) inside shading incubator set at $25^{\circ} \mathrm{C}$. Chemical compound fertilizer, mamekasei $\left(\mathrm{N}: \mathrm{P}_{2} \mathrm{O}_{5}: \mathrm{K}_{2} \mathrm{O}=\right.$ 3:10:10) $\left(2 \mathrm{~g} \cdot \mathrm{box}^{-1}\right)$ and lime $\left(3 \mathrm{~g} \cdot \mathrm{box}^{-1}\right)$ were well mixed with the soil before sowing. After 5 days of seeding, for RNA and protein analysis, each seedling (about $2 \mathrm{~g}$ ) was dipped in liquid nitrogen for a while and then all were stored at $-80^{\circ} \mathrm{C}$ until analysis.

\subsection{Treatments of Sucrose Starvation and Sucrose-Rich Medium}

Five-day-old cowpea seedlings were used for analyzing of protein. When the length of the hypocotyls reached between 6 and $8 \mathrm{~cm}$, cotyledons of the seedlings were removed to stop of the supply of nutrients. The seedlings were incubated at $25^{\circ} \mathrm{C}$ in a nutrient-rich, starvation or starvation-protease inhibitor medium, and harvested at intervals of 0,12 and 24 hours as described previously [15]. The nutrient-rich medium contained $10 \mathrm{mM}$ MES$\mathrm{KOH}, \mathrm{pH} 7.4,3 \%$ sucrose, $10 \mathrm{mM} \mathrm{KNO}, 2 \mathrm{mM}$ of

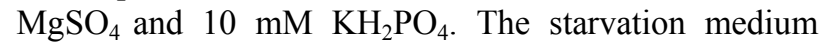
contained $10 \mathrm{mM}$ MES-KOH [pH 7.4], $10 \mathrm{mM} \mathrm{KCl}, 2$ $\mathrm{mM} \mathrm{MgSO}_{4}$, and $10 \mathrm{mM} \mathrm{KH}_{2} \mathrm{PO}_{4}$. Additionally, Starvation-protease inhibitor medium was supplemented with 1 $\mathrm{mM}$ phenylmethylsulfonyl fluoride (PMSF, a serine protease inhibitor), $10 \mu \mathrm{g} \cdot \mathrm{ml}^{-1}$ leupeptin (a cysteine protease inhibitor), $100 \mu \mathrm{M}$ E64-d (a membrane permeable cysteine protease inhibitor) (PEPTIDE Inc., Osaka, Japan) and $10 \mathrm{mM}$ quinacrine for alkalization of vacuolar $\mathrm{pH}$.

\subsection{Protein Preparation and Immunoblot}

To prepare protein samples for immunoblot, 5-day-old seedlings (about $1 \mathrm{~g}$ fresh weight) treated in nutrient-rich or starvation medium for 12 or 24 hour were harvested and homogenized on an ice-cooled mortor with liquid nitrogen and mixed with $3 \mathrm{~mL}$ of 1xTBScontaining 10 $\mathrm{mM}$ EDTA, 5\% glycerol, $1 \%$ Triton $\mathrm{X}-100,0.2 \% \beta$ mercaptoethanol, $1 \mathrm{mM}$ PMSF, $10 \mu \mathrm{g} \cdot \mathrm{mL}^{-1}$ leupeptin, and $1 \mathrm{mM}$ benzamidine. After centrifugation of the crude extracts at $5000 \times \mathrm{g}$ for $5 \mathrm{~min}$ at $4^{\circ} \mathrm{C}$, the resulting supernatant was subjected to SDS-polyacrylamide gel electrophoresis (SDS-PAGE) with 15\% acrylamide gel. Protein concentrations were determined with BioRad Protein Assay Kit (BioRad, Hercules, CA) using bovine serum albumin as standard. Immunoblot was carried out as described previously with minor modifications [16]. After SDS-PAGE with $15 \%$ acrylamide gels applied with each $50 \mu \mathrm{g}$ protein per lanes, separated polypeptides were electro-transferred to PVDF membranes (Millipore, Billerica, USA) in $25 \mathrm{mM}$ Tris base containing $0.05 \%$ SDS and $20 \%$ methanol at $10 \mathrm{~V} \cdot \mathrm{cm}^{-1}$ for 2 hours. After blocking with $1 \times$ TBS containing 3\% skim milk (TBS-milk) for 1 hour, the PVDF membrane was incubated in TBSmilk with anti-GmAtg8i specific antibody (dilution, 1/ 1000 ) and $0.05 \%$ Tween 20 for 2 hours at $4{ }^{\circ} \mathrm{C}$ and then in TBS-milk with horse raddish peroxidase-conjugated anti rabbit antibody (dilution, 1/5000 [v/v], GE Healthcare BioSciences Ltd., Piscataway, NJ) for 1 hour (Okuda et al., 2011). Immuno-reactive signals were visualized by ECL Plus Kit (GE Healthcare Bio-Sciences Ltd.) and FluorChem. 


\subsection{Protein Preparation and Immunoblot}

To prepare protein samples for immunoblot, 5-day-old seedlings (about $1 \mathrm{~g}$ fresh weight) treated in nutrient-rich or starvation medium for 12 or 24 hours were harvested and homogenized on an ice-cooled mortor with liquid nitrogen and mixed with $3 \mathrm{~mL}$ of 1xTBS containing 10 mM EDTA, 5\% glycerol, 1\% Triton X-100, 0.2\% $\beta$ mercaptoethanol, $1 \mathrm{mM}$ PMSF, $10 \mu \mathrm{g} \cdot \mathrm{mL}^{-1}$ leupeptin, and $1 \mathrm{mM}$ benzamidine. After centrifugation of the crude extracts at $5000 \times \mathrm{g}$ for $5 \mathrm{~min}$ at $4^{\circ} \mathrm{C}$, the resulting supernatant was subjected to SDS-polyacrylamide gel electrophoresis (SDS-PAGE) with 15\% acrylamide gel. Protein concentrations were determined with BioRad Protein Assay Kit (BioRad, Hercules, CA) using bovine serum albumin as standard. Immunoblot was carried out as described previously with minor modifications [16]. After SDS-PAGE with $15 \%$ acrylamide gels applied with each $50 \mu \mathrm{g}$ protein per lanes, separated polypeptides were electro-transferred to PVDF membranes (Millipore, Billerica, USA) in $25 \mathrm{mM}$ Tris base containing $0.05 \%$ SDS and $20 \%$ methanol at $10 \mathrm{~V} \cdot \mathrm{cm}^{-1}$ for 2 hour. After blocking with $1 \times$ TBS containing 3\% skim milk (TBS-milk) for 1 hour, the PVDF membrane was incubated in TBSmilk with anti-GmAtg8i specific antibody (dilution, 1/ 1000 ) and $0.05 \%$ Tween 20 for 2 hour at $4^{\circ} \mathrm{C}$ and then in TBS-milk with horse raddish peroxidase-conjugated anti rabbit antibody (dilution, 1/5000 [v/v], GE Healthcare Bio-Sciences Ltd., Piscataway, NJ) for 1 hour (Okuda et al. 2011). Immuno-reactive signals were visualized by ECL Plus Kit (GE Healthcare Bio-Sciences Ltd.) and FluorChem.

\section{Results}

\subsection{Sucrose Starvation Induces Autophagy and the Expression of ATGs}

A phylogenic analysis of deduced amino acid sequences of $A T G 8 \mathrm{c}$ and ATG8i homologs of Arabidopsis thaliana, soybean and cowpea showed that AtATG8i, GmATG8i and $V u A T G 8 i$ (tentative consensus number, TC11912) are classified into a higher plant specific clade of $A T G 8$ subfamilies while yeast $A P G 8, A t A T G 8 c, G m A T G 8 \mathrm{c}$ and $V u A T G 8 c$ (TC3559) are grouped into another clade of $A T G 8$ subfamilies (Figure 1(a)). The predicted amino acid sequence of $V u A T G 8 i$ has similarities of $92 \%$ and $69 \%$ to those of $G m A T G 8 i$ and AtATG8i, respectively. The predicted amino acid sequences of $V u A T G 8 c$ has similarities of $92 \%$ and $87 \%$ to those of $G m A T G 8 c$ and AtATG8c, respectively. VuATG4 (FG847544) was identified to have the highest similarites of $86 \%$ and $55 \%$ in deduced amino acid sequences to GmATG4 (Glyma18g48380) and AtATG4a (At2g44140), respectively.

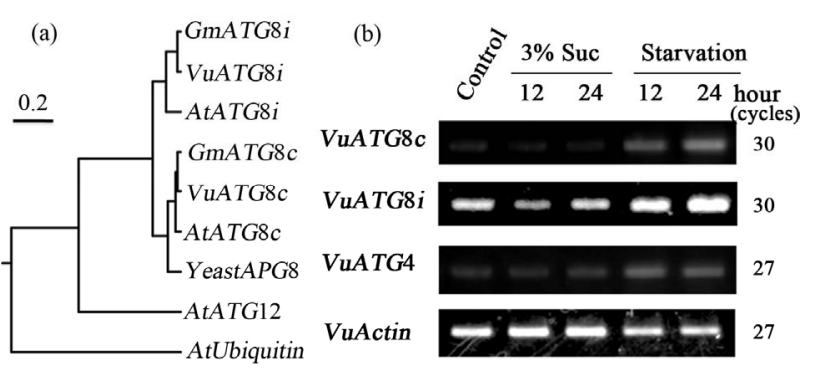

Figure 1. Effects of sucrose starvation on expression of autophagy-related genes. (a) A phylogenic tree of ATG8 homologs and ubiquitin-related proteins from cowpea and other organisms was constructed by ClustalW (UPGMA). The genes used were follows: VuATG8c, VuATG8i and VuATG4 (Table 1); GmATG8c (Glyma15g11510), GmATG8i (Glyma02g01180); AtATG8c (At1g62040), AtATG8i (At3g 15580), AtATG12 (At1g54210), AtUbiquitin (At4g05050); budding yeast APG8 (AY692870). Tentative consensus (TC) numbers of cowpea genes and gene index numbers of soybean and Arabidopsis were referred to TGI database (http://compbio. dfci.harvard.edu/tgi/plant.html) and Phytozome ver 9.0 (http:// www.phytozome.net/), respectively. (b) The expression profiles of cowpea ATG homologs in response to nutrient conditions are shown. Nutrient treatments and semi-quantitative RT-PCR was carried out at indicated numbers of thermal cycles as described in Materials and Methods. VuActin was used as a standard.

It was examined whether the expressions of $V u A T G 8 c, i$ and $V u A T G 4$ are regulated in response to various nutrient conditions. Sucrose starvation treatment on cowpea seedlings significantly enhanced the expression levels of $V u A T G 8 c$ and $V u A T G 4$ at 12 and 24 hour, while 3\% sucrose had little or marginal effect on the expression of those genes (Figure 1(b)). In all conditions, the expression levels of $V u A T G 8 i$ were relatively higher than those of $V u A T G 8 i$ and $V u A T G 4$. In contrast to VuATG$8 c$ and $V u A T G 4,3 \%$ sucrose treatment transiently suppressed the expression of $V u A T G 8 i$ at 12 hour. Sucrose starvation treatment slightly enhanced the expression levels of VuATG8. Previous studies with Arabidopsis and soybean revealed that the upregulation of $A T G 8 s$ and $A T G$-4 mRNA were accompanied with induction of autophagy in response to sucrose starvation stress [9, $15,17]$. The present data that sucrose starvation induced $V u A T G-8 c, i$ and VuATG4 is consistent to the previous observation on autophagy induction in Arabidopsis and soybean.

\subsection{Induction of Amino Acid Catabolism-Related Genes and a bZIP Transcription Factor}

Recently, low energy stresses such as reduced photosynthesis and/or sucrose starvation appeared to induce daramatic change of amino acid catabolism for asparagine 
Table 1. Gene specific oligo DNA primers used for RT-PCR and cloning.

\begin{tabular}{|c|c|c|c|}
\hline Gene name & $\begin{array}{c}\text { Accession } \\
\text { No. }\end{array}$ & $\mathrm{TC}^{*}$ & Primer sequences \\
\hline VuATG8c & AB779661 & TC3559 & $\begin{array}{c}\text { F-GTTGGATCCTCACAGATC } \\
\text { ATGGCCAAAAGC }\end{array}$ \\
\hline \multirow{3}{*}{ VuATG8i } & \multirow{3}{*}{ AB779662 } & \multirow{3}{*}{ TC11912 } & $\begin{array}{c}\text { R-ATGCGTCGACTATAGACA } \\
\text { ACAGAACTTGCA }\end{array}$ \\
\hline & & & $\begin{array}{c}\text { F-AACGGATCCATGGGAGG } \\
\text { AAGTTCAAGTTTC }\end{array}$ \\
\hline & & & $\begin{array}{c}\text { R-GAAGGTGCACCAGAACA } \\
\text { GAACAATGAGTGA }\end{array}$ \\
\hline \multirow[t]{2}{*}{ VuATG4 } & \multirow[t]{2}{*}{ AB779663 } & \multirow[t]{2}{*}{ FG847544 } & $\begin{array}{c}\text { F-GGCGGATCCCCGGTTGTC } \\
\text { TGCATTGAAGAT }\end{array}$ \\
\hline & & & $\begin{array}{l}\text { R-CAATGCCATGGATAAGA } \\
\text { GTTGCCAATCATC }\end{array}$ \\
\hline \multirow[t]{2}{*}{$V u A S N 1$} & \multirow[t]{2}{*}{ AB779664 } & \multirow[t]{2}{*}{ TC7410 } & $\begin{array}{l}\text { F-TGGGGTTTAGAAGCCAGA } \\
\text { GTACCA }\end{array}$ \\
\hline & & & $\begin{array}{c}\text { R-CAACTCCTAAGGGAGAA } \\
\text { ACCTCCA }\end{array}$ \\
\hline \multirow[t]{2}{*}{ VuProDH } & \multirow[t]{2}{*}{ AB779665 } & \multirow[t]{2}{*}{ TC15194 } & $\begin{array}{l}\text { F-TACCGGATCCATGCCAAA } \\
\text { GAGAGGTTGCATGCTC }\end{array}$ \\
\hline & & & $\begin{array}{l}\text { R-CCAAGTCGACATTAAAA } \\
\text { AACAGCAGCTTTTAGCCT }\end{array}$ \\
\hline \multirow[t]{2}{*}{ VuBCAT2 } & \multirow[t]{2}{*}{ AB779666 } & \multirow[t]{2}{*}{ TC3413 } & $\begin{array}{l}\text { F-GGAGGGATCCGCTCCGCT } \\
\text { CAACTTGCACGT }\end{array}$ \\
\hline & & & $\begin{array}{l}\text { R-CAATCGTCGACACCCTTG } \\
\text { CTTAATCTACTTCA }\end{array}$ \\
\hline \multirow[t]{2}{*}{ VubZIP53 } & \multirow[t]{2}{*}{ AB779667 } & \multirow[t]{2}{*}{ TC2820 } & $\begin{array}{c}\text { F-TCCCGGATCCTTGTCGGA } \\
\text { AATGGCATCGATTC }\end{array}$ \\
\hline & & & $\begin{array}{l}\text { R-TAAAGTCGACGGGGATG } \\
\text { AAATGGATCAACGCAG }\end{array}$ \\
\hline \multirow[t]{2}{*}{ VuActin } & \multirow[t]{2}{*}{ AF143208 } & \multirow[t]{2}{*}{-} & $\begin{array}{l}\text { F-GCGTGATCTCACTGATGC } \\
\text { CCTTAT }\end{array}$ \\
\hline & & & $\begin{array}{l}\text { R-AGCCTTCGCAATCCACAT } \\
\text { CTGTTG }\end{array}$ \\
\hline
\end{tabular}

${ }^{*}$ TC: tentative consensus number.

biosynthesis by inducing the expression of asparagine synthase (AtASN1), proline dehydrogenase1 (AtProDH), and branched chain aminoacid transaminase (AtBCAT2) $[18,19]$. Among cowpea homlog genes amino acid catabolic-related enzymes, VuASN1 (TC7410), VuProDH (TC15194) and $V u B C A T 2$ (TC3413) were identified to have the highest similarites of $82 \%, 62 \%$ and $62 \%$ in deduced amino acid sequences to AtASN1 (At3g47340), AtProDH (At3g30775) and AtBCAT2 (At1g10070), respectively. Heterodimers of bZIP1 and bZIP53 have been identified to be crucial transcriptional regulators in Asn, Pro and BCAA under sucrose starvation [20]. Among bZIP homolog genes in cowpea ESTs, VubZIP53 (TC2820) was identified to have the highest similarites of $51 \%$ and $26 \%$ in amino acid sequences to bZIP53 (At3g62420) and $b Z I P 1$ (At5g49450), respectively.

Sucrose starvation treatment significantly enhanced the expression of $V u A S N 1$ and $V u B C A T$ at 12 and 24 hour, while $3 \%$ sucrose treatment had little or marginal effects on that of $V u A S N 1$ and $V u B C A T$ (Figure 2). On the other hand, $3 \%$ sucrose treatment gradually repressed the expression of VuProDH at 12 and 24 hour, while sucrose starvation treatment slightly induced VuProDH. Sucrose starvation and 3\% sucrose treatmens both had little or marginal effects on the expression of VubZIP53, even though VubZIP53 is supposed to be a cowpea ortholog of AtbZIP53 which is induced under low energy stress [20].

\subsection{Effects of Sucrose Starvation on ATG8 Protein Levels in Cowpea Seedlings}

To analyze status of autophagy induction in cowpea seedling under various nutrient conditions, we analyzed the profiles of $A T G 8 i$-related protein in response to $3 \%$ sucrose and sucrose starvation treatment by antiGmATG8i specific antibody [15] (Figure 3). The previous studies revealed that sucrose starvation in the presence of leupeptin and E-64d significantly increases $A T G 8 i$ protein levels because degradation of autophagosomes composed of $A T G 8$ proteins in vacuole is efficiently inhibited by vacuolar proteases, leupeptin and E-64d [15]. Combination of sucrose starvation and protease inhibitors, leupeptin and E-64d, significantly increased the immunoreactive signals of $A T G 8 i$ protein at 12 and 24 hour, compared to sucrose starvation in the absence of leupeptin and E-64d (Figure 3). The significant difference of $A T G 8 i$ levels in sucrose-starved cowpea seedlings between the presence and the absence of protease inhibitors indicates that sucrose starvation stimulated autophagy leading to translocation of ATG8associating autophagosomes to vacuole. $3 \%$ sucrose treatment increased immunorective signals of $A T G 8 i$ related protein in cowpea seedling at 12 and 24 hour, while $3 \%$ sucrose treatment had little or marginal effects

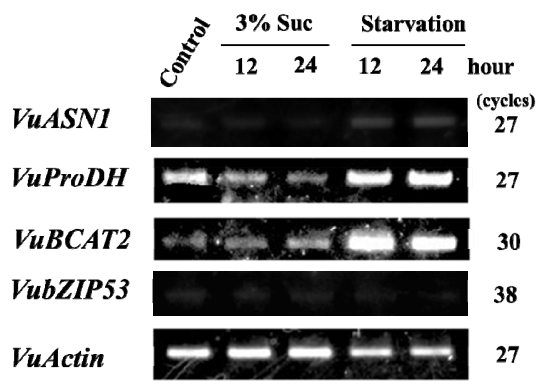

Figure 2. Effects of nutrient conditions on the expression of amino acid catabosim-related genes and bZIP. The expression of VuASN1, VuProDH, VuBCAT2 and VubZIP53 was analyzed. Nutrient treatments and semi-quantitative RTPCR was carried out at indicated numbers of thermal cycles as described in Materials and Methods. VuActin was used as a standard. 
on the expression of $V u A T G 8 i$ (Figures 1(b) and 3). It is supposed that the apparent increase of $A T G 8 i$ protein levels under 3\% sucrose resulted from reduction of degradation rate of $A T G 8$ proteins by suppression of autophagy.

\section{Discussion}

In higher plants, autophagy appeared to be induced by various environmental stresses to enhance nutrient capture, amino acid recycle and translocation of carbohydrates and nitrogen [21]. Several ATG mutants of Arabidopsis thaliana show early senescence phenotypes or high susceptibility to starvation stress [22,23]. It is reasonable that acceleration of autophagy and nitrogen metabolism in response to change of nutrient status play pivotal roles in senescence and nutrient translocation. The present data indicates that sucrose nutrient signals on cowpea seedlings have pleiotropic effects on autophagy and amino acid catabolism. Starvation signals enhanced the expressions of ATGS and amino acid catabolism-related genes (Figures 1(b) and 2). Thus, the expression of amino acid catabolism-related genes in response to sucrose starvation signal is regulated possibly by similar mechanisms to the expression of cowpea ATGs. In the other hand, sucrose feeding suppressed ATG8 degradation mediated by autophagy (Figure 3). It is possible that sucrose and amino acids play pivotal roles in the nutrient signal cascades regulating autophagy. Recently, It has been reported that low energy stress on Arabidopsis plant transcriptionally and translationally activates heterodimer bZIP trasnription factors, $b Z I P 1$ and bZIP53, which regulate the expression of $A S N 1, P r o D H$ and $B C A T 2$, via the promoter regions containing G-box, C-box and ACT motifs [20]. The expression of cowpea ZIP53 ortholog, VubZIP53, was not induced by sucrose starvation (Figure 2). However, the possibilities are not excluded that unidentified $b Z I P$ type transcription factors forming a heterodimer with VubZIP53 is induced by sucrose starvation or that VubZIP53 is translationaly activated by

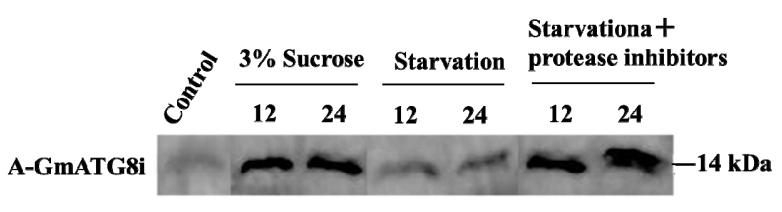

Figure 3. Immunoblot of ATG8i-related proteins in cowpea seedlings under various nutrient conditions. Cowpea seedlings were treated in $3 \%$ sucrose and sucrose starvation in the absence or the presence of protease inhibitors. Extracted proteins were subjected to SDS-PAGE and immunoblot by anti-ATG8i specific antibody as described in Materials and Methods. The immunoreactive signals of ATG8i-related polypeptides were detected by ECL at a relative molecular mass with about $14 \mathrm{kDa}$ as indicated. sucrose starvation. Recent studies unveiled that branched amino acid levels regulate autophagy by inactivation of TOR, which phosphorylates a crucial regulator complex, $A T G 1-A T G 13$ at upstream of autophagy induction [6]. It was reported that plant BCAT isozymes with different sub-cellular localization are regulated in complex manner by environmental stresses [24]. BCATs catalyse the final transamination step for synthesis of BCAA in plants and bacteria. The enzymes are also involved in the degradation of BCAA to provide an alternative carbon source under stress conditions in plants, animal and bacteria. Barley BCATs and Arabidopsis BCAT2 are induced by drought stress and sucrose starvation, respectively [19, 20]. The observation that sucrose starvation enhanced the expression of $V u B C A T 2$ prompted us to propose a hypothesis that an increased degradation of BCAA by BCAT results in inactivation of cowpea TOR, in tern, leading to induction of autophagy via $A T G 1-A T G 13$ cascade.

Regarding to drought tolerance of cowpea, drought stress enhances leaf senescence and seed maturation drastically in seed filling stage. Then, drought treatment transiently caused increase in sugar contents in leaves and then significantly enhanced sucrose transport from leaves to seeds [13]. In aspects of photoassimilate and sink-source balance, drought stress on cowpea caused significant reduction of photosynthesis by stomata closure and developing seeds accelerates uptake of carbohydrate from source organs, leading to occurrence of severe low energy stress in leaf tissues mimicking sucrose starvation. Our previous study indicates that increased uptake of photoassimilate by sink organs involved in autophagy induction, nitrogen translocation and leaf senescence of soybean at the pod filling stage [11]. In Arabidopsis, ASN1 mediated the biosynthesis of asparagine, which is used to store and transport nitrogen especially under stress conditions where carbon is limited [25]. In Arabidopsis, ProDH degradates the compatible osmolyte, proline, which is recycled to support carbon, nitrogen and energy demand under carbohydrate limitation [20]. ProDH is up- and down-regulated by hyposmotic condition and dehydration stress, respectively according to the role of proline as osmolyte [18]. Therefore, the expression of BCAT2, ASN1 and ProDH can be assumed to be the indicator of dramatic change of amino acid catabolism for carbon supplies and nitrogen remobilization. Based on the present results of sucrose starvetion experiments with cowpea seedling, it is conceivable that drought stress on cowpea at the seed filling stage, promotes asparagine biosynthesis and proline degradation via reduction of photoassimilate. Resultantly, drought stress accelerates nutrient translocation from senescing leaf to developing seed, rather than proline biosynthesis 
for maintaining drought tolerance of the leaf. To address mechanisms of rapid senescence of cowpea leaf at the seed filling stage in response to drought stress, we need to analyse $A T G s$ and amino acid catabolism-related genes in cowpea plant at the seed filling stage.

The present study reveal that sugar starvation signal on cowpea seedling stimulates both autophagy and amino acid catabolism possibly by transcriptional regulation of VuATGs, VuASN1, VuProDH and VuBCAT2. It still remains to be clarified how the sugar starvation signal regulates autophagy and the expression of those genes via bZIP53-like transcription factors and/or TOR-ATG1$A T G 13$ cascade. Increasing evidence suggests that the network of various nutrient signals, such as sugars and amino acids, regulate plant development, senescence and primary metabolisms. Therefore, identifications and molecular dissection of the signaling molecules involved in sugar signaling are important for research of autophagy and amino acid catabolism in higher plant.

\section{Acknowledgements}

This work was supported in part by Grant-in-Aids from the Ministry of Education, Sports, Culture, Science and Technology of Japan, No. 21658113 (to T. Yuasa) and No. 23380013 (to M. Iwaya-Inoue).

\section{REFERENCES}

[1] A. Ciechanover, "The Ubiquitin-Proteasome Pathway: On Protein Death and Cell Life," The EMBO Journal, Vol. 17, No. 24, 1998, pp. 7151-7160. doi:10.1093/emboj/17.24.7151

[2] Y. Kabeya, N. Mizushima, T. Ueno, A. Yamamoto, T. Kirisako, T. Noda, E. Kominami, Y. Ohsumi and T. Yoshimori, "LC3, a Mammalian Homologue of Yeast Apg8p, Is Localized in Autophagosome Membranes after Processing," The EMBO Journal, Vol. 19, No. 21, 2000, pp. 5720-5728. doi:10.1093/emboj/19.21.5720

[3] D. J. Klionsky, J. M. Cregg, W. A. Dunn Jr., S. D. Emr, Y. Sakai, I. V. Sandoval, A. Sibirny, S. Subramani, M. Thumm, M. Veenhuis and Y. Ohsumi, "A Unified Nomenclature for Yeast Autophagy-Related Genes," Developmental Cell, Vol. 5, No. 4, 2003, pp. 539-545. doi:10.1016/S1534-5807(03)00296-X

[4] Y. Ohsumi, "Molecular Dissection of Autophagy: Two Ubiquitin-Like Systems," Nature Reviews in Molecular Cell Biology, Vol. 2, No. 3, 2001, pp. 211-216. doi: $10.1038 / 35056522$

[5] T. Kirisako, Y. Ichimura, H. Okada, Y. Kabeya, N. Mizushima, T. Yoshimori, M. Ohsumi, T. Takao, T. Noda and Y. Ohsumi, "The Reversible Modification Regulates the Membrane-Binding State of Apg8/Aut7 Essential for Autophagy and the Cytoplasm to Vacuole Targeting Pathway," Journal of Cell Biology, Vol. 151, No. 2, 2000, pp. 263-276. doi:10.1083/jcb.151.2.263
[6] Y. Chen and D. J. Klionsky, "The Regulation of Autophagy-Unanswered Questions," Journal of Cell Science, Vol. 124, No. 2, 2011, pp. 161-170. doi: $10.1242 /$ jes.064576

[7] C. Robaglia, M. Thomas and C. Meyer, "Sensing Nutrient and Energy Status by SnRK1 and TOR Kinases," Current Opinion in Plant Biology, Vol. 15, No. 3, 2012, pp. 301307. doi:10.1016/i.pbi.2012.01.012

[8] S. Slavikova, G. Shy, Y. Yao, R. Glozman, H. Levanony, S. Pietrokovski, Z. Elazar and G. Galili, "The Autophagy-Associated Atg8 Gene Family Operates Both Under Favorable Growth Conditions and under Starvation Stresses in Arabidopsis Plants," Journal of Experimental Botany, Vol. 56, No. 421, 2005, pp. 2839-2849. doi:10.1093/jxb/eri276

[9] T. L. Rose, L. Bonneau, C. Der, D. Marty-Mazars and F. Marty, "Starvation-Induced Expression of AutophagyRelated Genes in Arabidopsis," Biology of the Cell, Vol. 98, No. 1, 2006, pp. 53-67. doi:10.1042/BC20040516

[10] H. Rouached, A. B. Arpat and Y. Poirier, "Regulation of Phosphate Starvation Responses in Plants: Signaling Players and Cross-Talks," Molecular Plant, Vol. 3, No. 2, pp. 288-299. doi: $10.1093 / \mathrm{mp} / \mathrm{ssp} 120$

[11] M. P. S. H. Nang, T. Yuasa, Y. Ishibashi, M. Okuda, H. Tanigawa, S. H. Zheng and M. Iwaya-Inoue, "Leaf Senescence of Soybean at Reproductive Stage Is Associated with Induction of Autophagy-Related Genes, GmATG8c, GmATG8i and GmATG4," Plant Production Science, Vol. 14, No. 2, 2011, pp. 141-147. doi:10.1626/pps.14.141

[12] J. D. Ehlers and A. E. Hall, "Cowpea (Vigna unguiculata L. Walp.)," Field Crops Research, Vol. 53, No. 1, 1997, pp. 187-204. doi:10.1016/S0378-4290(97)00031-2

[13] M. Arimura, Y. Hashiguchi, M. Imamura, T. Yamaguchi, T. Yuasa and M. Iwaya-Inoue, "Effect of Drought Stress on Carbohydrate Status in Leaves and Seeds of Cowpea during Pod Filling Stage," Japanese Journal of Crop Science, Vol. 226, No. 2, 2008, pp. 260-261.

[14] T. Sakamoto, Y. Hashigchi, E. Kurauchi, M. Imamura, Y. Ishibashi, S. Muranaka, T. Yuasa and M. Iwaya-Inoue, "Causative Factors of Decreasing Flower Number in Cowpea under Drought Stress during Flowering Stage," Crybiology and Cryotechnology, Vol. 58, No. 1, 2012, pp. 81-85.

[15] M. P. S. H. Nang, H. Tanigawa, Y. Ishibashi, S. H. Zheng, T. Yuasa and M. Iwaya-Inoue, "Nutrient Starvation Differentially Regulates the Autophagy-Related Gene GmATG8i in Soybean Seedlings," Plant Biotechnology, Vol. 26, No. 3, 2009, pp. 317-326. doi:10.5511/plantbiotechnology.26.317

[16] J. Nakamura, T. Yuasa, H. T. Tran, K. Harano, S. Tanaka, T. Iwata, T. T. Phan and M. Iwaya-Inoue, "Rice Homologs of Inducer of CBF Expression (OsICE) Are Involved in Cold Acclimation," Plant Biotechnology, Vol. 28, No. 3, 2011, pp. 303-309. doi:10.5511/plantbiotechnology.11.0421a

[17] M. Okuda, M. P. Nang, K. Oshima, Y. Ishibashi, S. H. Zheng, T. Yuasa and M. Iwaya-Inoue, "The Ethylene Signal Mediates Induction of GmATG8i in Soybean Plants 
under Starvation Stress," Bioscience Biotechnology Biochemistry, Vol. 75, No. 7, 2011, pp. 1408-1412. doi:10.1271/bbb.110086

[18] K. Nakashima, R. Satoh, T. Kiyosue, K. YamaguchiShinozaki and K. Shinozaki, "A Gene Encoding Proline Dehydrogenase Is Not Only Induced by Proline and Hypoosmolarity, but Is Also Developmentally Regulated in the Reproductive Organs of Arabidopsis," Plant Physiology, Vol. 118, No. 4, 1998, pp. 1233-1241. doi:10.1104/pp.118.4.1233

[19] M. Malatrasi, M. Corradi, J. T. Svensson, J. Close, M. Gull and N. Marmiroli, "A Branched-Chain Amino Acid Aminotransferase Gene Isolated from Hordeum Vulgare Is Differentially Regulated by Drought Stress," Theoretical Applied Genetics, Vol. 113, No. 6, 2006, pp. 965-976. doi:10.1007/s00122-006-0339-6

[20] K. Dietrich, F. Weltmeier, A. Ehlert, C. Weiste, M. Stahl, K. Harter and W. Dröge-Laser, "Heterodimers of the Arabidopsis Transcription Factors bZIP1 and bZIP53 Reprogram Amino Acid Metabolism during Low Energy Stress," Plant Cell, Vol. 23, No. 1, 2011, pp. 381-395. doi:10.1105/tpc.110.075390

[21] A. Guiboileau, K. Yoshimoto, F. Soulay, M. P. Bataille, J. C. Avice and C. Masclaux-Daubresse, "Autophagy Ma- chinery Controls Nitrogen Remobilization at the WholePlant Level under Both Limiting and Ample Nitrate Conditions in Arabidopsis," New Phytologist, Vol. 194, No. 3, 2012, pp. 732-740. doi:10.1111/j.1469-8137.2012.04084.x

[22] J. H. Doelling, J. M. Walker, E. M. Friedman, A. R. Thompson and R. D. Vierstra, "The APG8/12-Activating Enzyme APG7 Is Required for Proper Nutrient Recycling and Senescence in Arabidopsis thaliana," Journal of Biological Chemistry, Vol. 277, No. 36, 2002, pp. 3310533114. doi:10.1074/jbc.M204630200

[23] K. Yoshimoto, H. Hanaoka, S. Sato, T. Kato, S. Tabata, T. Noda and Y. Ohsumi, "Processing of ATG8s, UbiquitinLike Proteins, and Their Deconjugation by ATG4s Are essential for Plant Autophagy," Plant Cell, Vol. 16, No. 11, 2004, pp. 2967-2983. doi:10.1105/tpc.104.025395

[24] S. Binder, "Branched-Chain Amino Acid Metabolism in Arabidopsis thaliana," The Arabidopsis Book, Vol. 8, 2010, Article ID: e0137. doi:10.1199/tab.0137

[25] H. M. Lam, S. S. Peng and G. M. Coruzzi, "Metabolic Regulation of the Gene Encoding Glutamine-Dependent Asparagine Synthetase in Arabidopsis thaliana," Plant Physiology, Vol. 106, No. 4, 1994, pp. 1347-1357. doi:10.1104/pp.106.4.1347 